\title{
Simulation and Analysis of Transverse Interface Cracking of Carbon Fiber Reinforced Composite
}

\author{
Dan $\mathrm{Hu}^{1, a}$, Chenghong Duan ${ }^{1, \mathrm{~b}}$ \\ ${ }^{1}$ CAE, Beijing University of Chemical Technology, Beijing, 100029, China \\ ahudannn@163.com, bduanchenghong@163.com
}

Keywords: XFEM, Surface-based cohesive behavior, interface, crack

\begin{abstract}
The Carbon fiber composite material has been widely used for its high specific strength, stiffness and many other excellent properties. But for the unidirectional carbon fiber composite, the transverse performance is much poorer than the longitudinal performance. Under the transverse load, interface debonding and matrix cracking tend to occur. In this paper the software ABAQUS was used to model the mechanical behavior of the carbon fiber composite under the transverse load, the extended finite element method (XFEM) and surface-based cohesive behavior were applied to simulate the interface and matrix transverse cracking in the composite. This simulation method laid a foundation for further studies on the interface and transverse property of the carbon fiber composite.
\end{abstract}

\section{Introduction}

The carbon fiber composite materials have been widely used in all kinds of areas, such as the automobile, aerospace and sports equipment, for their higher specific strength, stiffness and outstanding designability [1]. However the transverse mechanical property of the carbon fiber composite is weak. Under the transvers load, the reinforcing effect of the fiber is limited. As the link between the fiber and matrix, the interface is the key factor to the composite transverse performance.

Scholars around the world has made many contributions to the studies on the interface and transverse properties of the carbon fiber composites. The early studies focused on the tests and experiments, Andreas Domnanovich et al proposed a carbon fiber bundle pull-out test method to measure the interface property parameters of the $\mathrm{C} / \mathrm{C}$ composite [2], and now it has been widely used. Numerical simulation achievements have emerged in endlessly with the rapid development of the computer technology. Falk K. Wittel et al studied the transverse cracking process of the $90^{\circ}$ layer in the [0/90]s laminate with the disordered spring network model. This method accounted for the complicated load stress fields around failed regions, and it captured the gradual activation of the relevant failure mechanisms and their interactions during the fracture process [3]. In order to be closer to the real situation, Jiahai Lu et al [4] built the finite element model containing fibers, matrix and interfaces, simulated the interface with cohesive elements, to predict the transverse isotropic properties of the unidirectional carbon fiber composite.. Based on the micromechanics, Xiaoqiang Wang et al [5] studied the interface property's effects on the composite transverse effective property and damage process with the representative volume element model. In this paper, XFEM and Surface-based Cohesive Behavior in ABAQUS were applied to simulate the transverse cracking process of the carbon fiber composite.

\section{Finite element model}

Usually under a transverse load large enough, interface and matrix cracking will occur to the carbon fiber composite. In order to analyze the loading process, XFEM and Surface-based cohesive behavior provided by ABAQUS are combined in this paper.

The crack growth in the matrix can be simulated with XFEM. Compared with the traditional FEM, more additional functions have been added to it, such as the jump function that describes the displacement discontinuity, crack-tip function, incremental displacement field function, etc. In the XFEM, the element mesh and crack are independent of each other, so there is no need to reconstruct 
the mesh while the crack propagates, and a preset crack becomes unnecessary, moreover a crack can propagate along any path in the material [6].

But the XFEM cannot simulate the interface crack conveniently, so here comes to the surface-based cohesive behavior. The surface-based cohesive behavior is a kind of contact form, however it provides a simple way to model the cohesive interface. The cohesive surface behavior can be established readily by defining contact pair in the ABAQUS, and the interface properties are described by the contact property parameters.

Surface-based cohesive behavior has several stress-displacement modes, i.e. bilinear, exponential and tabular mode. Bilinear mode is chosen here, and it can be expressed as:

$$
\sigma= \begin{cases}\frac{\sigma_{0}}{\delta_{0}} \delta & \left(\delta \leq \delta_{0}\right) \\ \sigma_{0} \frac{\delta_{f}-\delta}{\delta_{f}-\delta_{0}} & \left(\delta_{f} \geq \delta>\delta_{0}\right) \\ 0 & \left(\delta \geq \delta_{f}\right)\end{cases}
$$

Where $\sigma_{0}$ is the initial failure stress, $\delta_{0}$ is the initial failure displacement, $\delta_{\mathrm{f}}$ is the complete failure displacement. When: $\delta<\delta_{0}$, the interface is in the elastic state; $\delta_{0}<\delta<\delta_{\mathrm{f}}$, it is in the damage evolution stage, and some relevant properties will be degraded; $\delta \geq \delta_{\mathrm{f}}$, the interface is completely damaged.

The initial damage criterion applied here is quadratic stress criterion, it can be expressed as:

$$
\left(\frac{<\sigma_{n}>}{\sigma_{n}^{\max }}\right)^{2}+\left(\frac{\sigma_{s}}{\sigma_{s}^{\max }}\right)^{2}+\left(\frac{\sigma_{t}}{\sigma_{t}^{\max }}\right)^{2}=1
$$

Where, $\sigma_{\mathrm{n}}{ }^{\max }, \sigma_{\mathrm{s}}{ }^{\max }, \sigma_{\mathrm{t}}{ }^{\max }$ are critical normal contact stress, critical shear contact stress along the first shear direction and critical shear contact stress along the second shear direction, their values in this paper are all 57.7MPa.

As to the damage evolution stage, the energy-based evolution criterion is applied, the fracture energy is $36.7 \mathrm{~J} / \mathrm{m}^{2}$ here. Similar to the stiffness degradation coefficient of cohesive element that describes the material's degradation in the damage evolution stage, the degradation coefficient $\mathrm{D} \in$ $[0,1]$ has been introduced into the cohesive surface behavior. $D=0$ means no damage, $D=1$ means completely damage and crack. See the Eq.3:

$$
\begin{aligned}
& \sigma_{n}= \begin{cases}(1-D) \sigma_{n}^{0} & \sigma_{n}^{0} \geq 0 \\
\sigma_{n}^{0} & \sigma_{n}^{0} \leq 0 \text { (no damage to compressive stiffness) }\end{cases} \\
& \sigma_{s}=(1-D) \sigma_{s}^{0} \\
& \sigma_{t}=(1-D) \sigma_{t}^{0}
\end{aligned}
$$

Where, $\sigma_{\mathrm{n}}^{0}, \sigma_{\mathrm{s}}{ }^{0}, \sigma_{\mathrm{t}}{ }^{0}$ are contact stress components predicted by the elastic traction-separation behavior for the current separations without damage.

Assume the carbon fibers are squarely distributed in the matrix, take the representative element as shown in Fig. 1. Where $\mathrm{L}=8.408 \mu \mathrm{m}$, diameter of the carbon fiber $\mathrm{R}=3 \mu \mathrm{m}$.

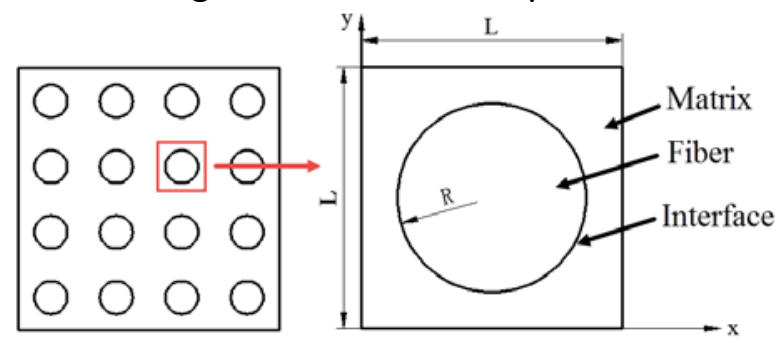

Fig. 1 Model selection

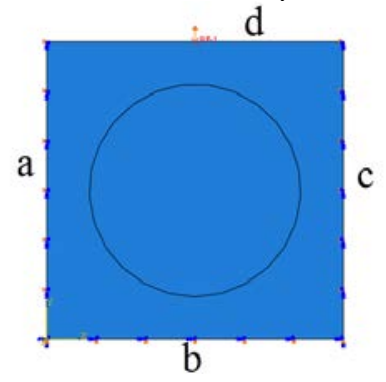

Fig.2 Finite element model

The matrix and carbon fiber were built respectively as two parts in the ABAQUS, then they were assembled to form the surface-based cohesive behavior contact. The geometry model was meshed 
with element type SR4 in SWEEP method. As for boundary conditions, the normal displacements of edge a, b and c were constraint, and upward displacement load was applied to the edge d. See Fig.2.

The material property parameters are list in Table.1. Generally it is very rare that the carbon fiber cracks transversely, and the carbon fiber strength is much higher than the matrix.

Table. 1 Material property parameters

\begin{tabular}{|c|c|c|c|c|}
\hline & \multicolumn{2}{|c|}{ Carbon fiber } & \multicolumn{2}{|c|}{ Matrix } \\
\hline $\begin{array}{l}\text { Young's modulus } \\
\text { (GPa) }\end{array}$ & $\begin{array}{l}E_{f 1} \\
E_{f 2}\end{array}$ & $\begin{array}{l}294 \\
18.4\end{array}$ & $\mathrm{E}_{\mathrm{m}}$ & 3.4 \\
\hline $\begin{array}{l}\text { Shear modulus } \\
\quad(\mathrm{GPa})\end{array}$ & $\begin{array}{l}\mathrm{G}_{\mathrm{f} 12} \\
\mathrm{G}_{\mathrm{f} 23}\end{array}$ & $\begin{array}{c}12 \\
6.39\end{array}$ & $\mathrm{G}_{\mathrm{m}}$ & 1.3 \\
\hline $\begin{array}{c}\text { Poisson's ratio } \\
\text { Transverse strength (MPa) }\end{array}$ & $\mathrm{u}_{\mathrm{f} 12}$ & 0.2 & $\mathrm{u}_{\mathrm{m}}$ & 0.35 \\
\hline
\end{tabular}

\section{Result analysis}

Fig.3 shows the Mises stress contour of the loading process.

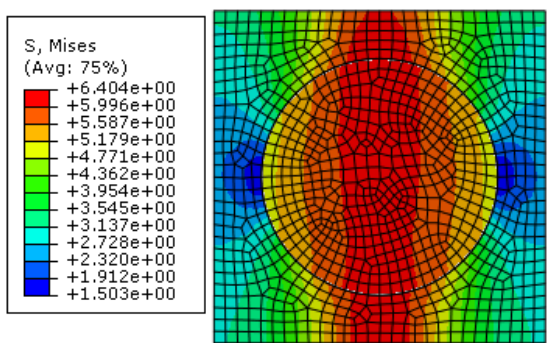

(a) Time $=0.0033$

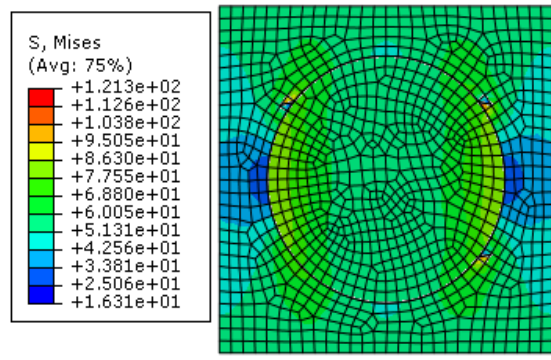

(c) Time $=0.058$

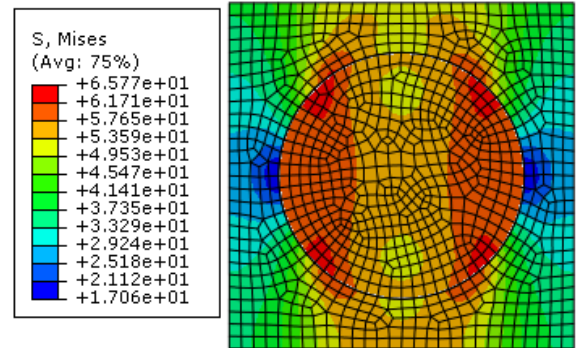

(b)Time $=0.039$

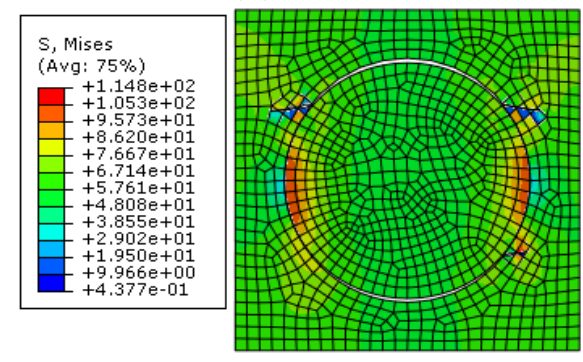

(d)Time $=0.078$

Fig.3 Stress contour of the loading process

As shown in Fig.3, when time $=0.0033$ the overall stress level is low, and the load is not high enough to make the interface deform observably, the fiber and matrix bond tightly. The high stress zone goes through the model from the top to the bottom, and the stress level of the fiber is relatively higher. That is to say in the elastic stage of the composite, the carbon fiber plays a main role of bearing load and its strengthening effect is obvious. When time $=0.039$, the overall stress level rise. In order to coordinate the deformation between the fiber and matrix, the interface's deformation along the load direction increases, meanwhile the high stress zone transfers to the left and right sides of the fiber. When time $=0.058$, the interface at top and bottom of the fiber begins to crack, until time $=0.078$ the cracks propagate into the matrix on both side of the fiber, the high stresses concentrates in the fiber near the uncracking interface. The final crack path is shown in Fig.4, in the finite element model the matrix above the fiber has been pulled apart, and it can be seen that the simulation result is similar to the real situation. 

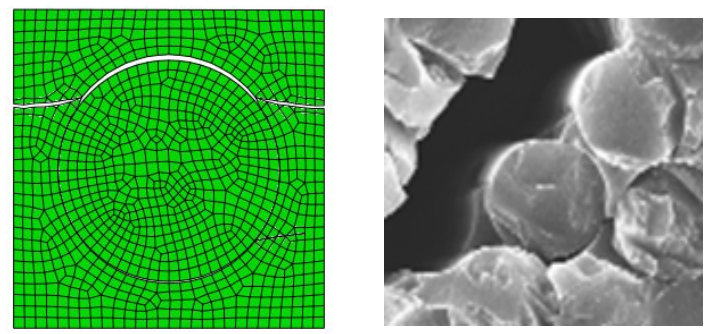

Fig.4 Simulation crack and real crack in the composite

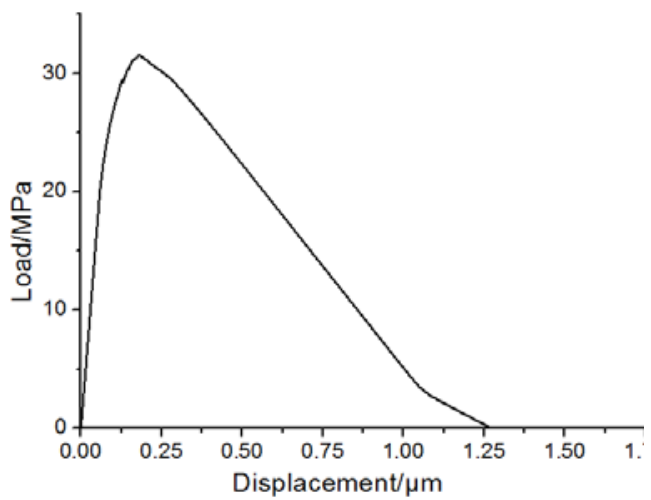

Fig.5 Load-displacement curve of the loading process

Fig. 5 shows the load-displacement curve of the entire loading process. The process can be divided into three stages, those are linear elastic, yielding and unloading stage. When the load reaches about 20MPa the elastic stage ends, this point is similar to the yielding point of metal material, after that the slope of the curve begins to decrease. The interface deformation continues to increase, until damage and crack occurs. The model loses its carrying capacity after the maximum load point 31.6MPa.

\section{Conclusion}

In conclusion, combining the XFEM and surface-based cohesive behavior as a simulation method is able to model every stage of the carbon fiber composite's performance under the transverse load. Especially it presents the interface and matrix cracking process detailedly and visually, the deformation and crack path are approximate to the real situation. This method lays a foundation for further studies on the interface and transverse mechanical behavior of the carbon fiber composite.

\section{References}

[1] Branislav Duleba, L'udmila Dulebová, Emil Spišák. Simulation and evaluation of carbon/epoxy composite systems using FEM and tensile test J. Procedia Engineering. 96 (2014) 70-74.

[2] Andreas Domnanovich, Herwig Peterlik, Karl Kromp. Determination of interface parameters for carbon/carbon composites by the fiber-buddle pull-out test J. Composites Science Technology. 56 (1996) 1017-1029.

[3] Falk K. Wittel, Jan Schulte-Fischedick, Ferenc Kun c, Bernd-H. Kroplin €, Martin Frieß. Discrete element simulation of transverse cracking during the pyrolysis of carbon fibre reinforced plastics to carbon/carbon composites J. Computational Materials Science. 28 (2003) 1-15.

[4] Jiahai Lu, Ping Zhu, Qinghui Ji, Qi Feng, Jian He. Identification of the mechanical properties of the carbon fiber and the interphase region based on computational micromechanics and Kriging metamodel J. Computational Materials Science. 95 (2014) 172-180.

[5] Xiaoqiang Wang, Jifeng Zhang, Zhenqing Wang, Song Zhou, Xinyang Sun. Effects of interphase properties in unidirectional fiber reinforced composite materials J. Materials and Design. 32 (2011) 3486-3492.

[6] Kästner M, Müller S, Ulbricht V. XFEM modelling of inelastic material behaviour and interface failure in textile-reinforced composites J. Procedia Materials Science. 2 (2013) 43-51. 